Tang and He Afr J Tradit Complement Altern Med. (2013) 10(4):12-17

http://dx.doi.org/10.4314/ajtcam.v10i4.3

\title{
TREATMENT OF D-GALACTOSE INDUCED MOUSE AGING WITH LYCIUM BARBARUM POLYSACCHARIDES AND ITS MECHANISM STUDY
}

Tao Tang and Bixiu He*

\author{
Department of Geriatrics, Xiangya Hospital, Central South University, Changsha 410008, Hunan, \\ China. \\ *Email: hebixiu000@163.com
}

\begin{abstract}
We evaluated the effects of Lycium barbarum polysaccharides LBP) on D-galactose aging model mouse, and explored its possible mechanism. Kunming mice were randomly divided into the control group, the model group, the high-dose LBP group, and the low-dose LBP group. Except the control group, D-galactose was used for modelling. The drug was administrated when modelling. Mouse behavioural, learning and memory changes were observed, and the contents of lipid peroxidation (LPO), lipofuscin (LF) and monoamine oxidase B (MAO-B) in mouse brain tissue and the weight of immune organs were measured after 6 weeks. Compared with the control group, mouse weight gain in the model group reduced significantly. Compared with model group, after mice drank LBP, the times of electric shock was less than aging mice (in which, the high-dose LBP group, $\mathrm{P}<0.05)$, and electric shock incubation period was longer $(\mathrm{P}<0.01)$. On Day 45 after modelling and drug administration, the contents of LPO, LF and MAO-B in mouse brain tissue in the model group increased significantly, while those in the drug administration groups decreased significantly. The thymus index in the aging model group decreased significantly; the thymus index and the spleen index in the high-dose LBP group and the low-dose LBP group rebounded significantly $(\mathrm{P}<0.01)$. We concluded that LBP has an anti-aging effect on D-galactose induced aging model mouse, and its mechanism may be related with the alleviation of glucose metabolism disorder and the resistance of the generation of lipid peroxide and other substances, which damage cell membrane lipid.
\end{abstract}

Key words: LBP; D-galactose; anti-aging

\section{Introduction}

Barbary wolfberry fruit is the dried ripe fruit of Lycium barbarum L. (State Pharmacopeia Committee., 2010), with immunity enhancing, liver protecting, blood sugar lowering, anti-tumour, blood pressure lowering, antioxidant, and anti-aging effects (Kim et al., 1997; Kim et al., 2000; Peng \& Tian., 2001; Leung et al., 2001; Toyoda-Ono et al., 2004) and other functions, and is a traditional precious Chinese herbal medicine of long history. The chemical composition analysis on barbary wolfberry fruit shows that it mainly contains polysaccharides, amino acids, trace elements, vitamins, taurine, alkaloids, volatile oil and other chemical ingredients (Zhou \& Li., 2009), in which, lycium barbarum polysaccharides (LBP), due to its significant pharmacological activity, becomes a research hotspot. This paper starts from the metabolism theory of aging, and uses D-galactose aging mouse model (Miao., 2002) to study the anti-aging effect of LBP and to preliminarily explore its anti-aging mechanism.

\section{Experimental materials}

\section{Drug and reagents}

Drugs and reagents used for the study include the following: barbary wolfberry from Zhongning County, Ningxia 
http://dx.doi.org/10.4314/ajtcam.v10i4.3

Province, China (which was identified by Professor Li Shixia of Central South University and deposited in the pharmacy centre); TBA (Shanghai Yongzheng Chemical Co., Ltd); TEP (Shanghai Weijing Biotechnology Co., Ltd); quinine sulphate (Shanghai Huyu Biology Co., Ltd.); benzylamine (Tianjin Jinhai Chemical Co., Ltd); perchloric acid (Tianjin Xinyuan Chemical Co., Ltd).

\section{Main instruments}

The main instruments for the study include the following: KQ-5200 ultrasonic extractor (Kunshan Ultrasonic Instrument Co., Ltd.); DT-200 jumping apparatus (Chengdu Taimeng Technology Co., Ltd); UV2100 ultraviolet and visible spectrophotometer (USA UNICO (Shanghai) Instrument Co., Ltd.); fluorescence spectrophotometer (Shanghai Jianglai Experimental Device Co., Ltd.); electric homogeniser (Zhenjiang Kemi Instrument \& Meter Co., Ltd); GL-21M high-speed refrigerated centrifuge (Changsha Xiangyi Centrifuge Instrument Co., Ltd.)

\section{Experimental animals}

Kunming mice are of clean grade, female, body weight $26-32 \mathrm{~g}$

\section{Preparation of LBP}

Referring to the methods in literature (Ayiguli et al., 2007) and making appropriate improvements, the detailed method is described below. Weigh appropriate amount of dried barbary wolfberry, make twice backflow extractions with chloroform: methanol (2:1) in $50^{\circ} \mathrm{C}$ water bath, 2 hours each time, discard the liquid, make ultrasonic extraction with the residue and $80 \%$ ethanol for three times, $20 \mathrm{~min}$ each time, filter and discard the filtrate, make ultrasonic extractions with the residue and water for three times, $20 \mathrm{~min}$ each time, filter, make low temperature concentration to the combined filtrate, add $95 \%$ ethanol, stand at low temperature for $24 \mathrm{~h}$, and get the crude polysaccharide extract after washing the extracted and filtered solids with absolute ethanol and acetone and vacuum drying.

\section{Animal grouping and drug administration}

48 mice were randomly divided into four groups, namely the control group, the model group, the high-dose LBP group and the low-dose LBP group ( $\mathrm{n}=12)$. Drinking method was adopted for drug administration, with the dose of $1 \mathrm{~g} /(\mathrm{kg} \bullet \mathrm{d})$ and $3 \mathrm{~g} /(\mathrm{kg} \bullet \mathrm{d})$. The model group and the control group used the equivalent amount of reference liquid. At the same time of drug administration, the mice in the model group and the drug administration groups were subcutaneously injected with $50 \%$ D-galactose in neck and back daily, $0.5 \mathrm{ml}$ per mouse, for continuous 6 weeks, and the mice in the control group were subcutaneously injected with equivalent amount of saline. Injection meets the sterile requirements. The indicators were determined at 6 weeks after modelling and drug administration.

\section{General behaviour observation}

During the experiment, we daily observed the general behavioural changes of mice, including feeding, activity, hair colour and others. We weighed the body weight of mice every 5 days, and observed the weight changes in all the groups. 


\section{Jumping test (Xu et al., 2003)}

6 weeks after modelling and drug administration, the mice were put into the response box to adapt for a few minutes, and then it was electrified. The mice would jump onto the platform to avoid shock; $5 \mathrm{~min}$ training for each mouse was observed. After $24 \mathrm{~h}$, we put the trained mice onto the platform and started the test. We recorded the time of the first jump off the platform, denoted as electric shock incubation period, and recorded the total number of electric shocks within $5 \mathrm{~min}$. While testing, if the time of staying on the platform exceeded $5 \mathrm{~min}$, the incubation period was deemed as $5 \mathrm{~min}$. (Operating parameter: $28 \mathrm{~V}$; time: $5 \mathrm{~min}$ )

\section{Determination of LPO, LF and MAO-B in brain tissue}

After the behaviour experiment and at 45 days after modelling and drug administration, the mice were decapitated, and the whole brain (cerebellum removed) was taken out quickly and separated at the mid sagittal suture, one half for the determination of LPO and LF contents, and the other half for the determination of MAO-B activity. LPO content was determined by TBA reaction colorimetry; LF content was determined by the Sohal method. We weighed 100mg brain, added $2 \mathrm{ml}$ chloroform - methanol (2:1) extract, homogenised and filtered. We washed the residue with the extract, combined the filtrates, added the extract to $5 \mathrm{ml}$, and measured the fluorescence intensity on a fluorescence spectrometer. Emission wavelength was $435 \mathrm{~nm}$ and excitation wavelength was $365 \mathrm{~nm}$. MAO-B activity was measured by the method of benzylamine. We took appropriate amount of fresh brain tissue, added 10 times of volume of pre-cooled $0.2 \mathrm{~mol} / \mathrm{L}$ phosphate buffer ( $\mathrm{pH}$ 7.4), homogenised in a glass homogeniser in ice bath, and then made ultrasonic extraction for 20 seconds twice and at an interval of 30 seconds, centrifuge at $1000 \times \mathrm{g}$ and $0^{\circ} \mathrm{C}$ for 10 minutes. We discarded the sediment, centrifuged the supernatant at $17000 \times \mathrm{g}$ and 4 for 30 minutes, and re-suspended the sediment with $0.2 \mathrm{~mol} / \mathrm{L}$ phosphate buffer. We took $0.5 \mathrm{ml}$ above enzyme solution, added $0.3 \mathrm{ml} 8 \mathrm{mmol} / \mathrm{L}$ benzylamine, and also added $2.5 \mathrm{ml} 0.2 \mathrm{~mol} / \mathrm{L}$ phosphate buffer (pH7.4). The blank tube was only added with $3.0 \mathrm{ml} 0.2 \mathrm{~mol} / \mathrm{L}$ phosphate buffer, and keep warm in a 37 water bath oscillator for 3 hours, mixing once every 15 minutes. Then, we added $0.3 \mathrm{ml} 60 \%$ perchloric acid to terminate the reaction, added $4.0 \mathrm{ml}$ cyclohexane for mixing and extraction, centrifuged at 3000 $/ \mathrm{min}$ for 10 minutes, and took the supernatant to measure the A value at $242 \mathrm{~nm}$. The difference between the groups was compared with the absolute hourly absorbance as the indicator $(\triangle \mathrm{A} / \mathrm{h})$.

\section{Determination of immune organ mass index}

Mice were weighed before being killed, the thymus and spleen were weighed on an electronic tissue scale after cleaning, and the thymus index and spleen index were calculated.

\section{Statistical methods}

SPSS11.0 software was adopted to make the statistical analysis on the experimental results. T test was adopted for intergroup mean comparison. One-way analysis of variance was adopted for multi-group mean comparison.

\section{Results}

Results of general behaviour observation

Before modelling, the mice in all the groups had normal activity, sensitive response, bright hair colour and normal diet. Compared with the control group 1 week after modelling, the mice in the model group had slow response, somnolence, 
http://dx.doi.org/10.4314/ajtcam.v10i4.3

decreased diet, reduced body weight, less activity, and dried and dull hair. The abnormal changes in the high-dose LBP group and the low-dose LBP group were remitted at various degrees.

Body weight change after 6 weeks is shown in Table 3-1.

Table 1: Effect of LBP on body weight of D-galactose-caused aging mice $(n=12)(\bar{X} \pm S)$

\begin{tabular}{ll}
\hline Group & Body weight change after 6 weeks $(\mathrm{g})$ \\
\hline The control group & $6.4 \pm 1.07$ \\
The model group & $1.3 \pm 0.75^{\bowtie}$ \\
The low-dose LBP group & $3.1 \pm 1.01^{*}$ \\
The high-dose LBP group & $4.6 \pm 1.08^{*}$ \\
\hline
\end{tabular}

${ }^{\diamond}$ Compared with the control group, $\mathrm{P}<0.01 ;{ }^{*}$ "Compared with the model group, $\mathrm{P}<0.01$

\section{Results of jumping test}

The results of the jumping test are shown in Table 3-2. The results show that after the mice drank LBP, the number of electric shocks are less than that for aging mice (the high-dose LBP group, $\mathrm{P}<0.05$ ), and the electric shock incubation period prolongs $(\mathrm{P}<0.01)$, indicating that mouse memory and learning ability are enhanced and LBP has the effects of improving animal learning and memory behaviour.

Table 2: Results of jumping test $(\overline{\mathrm{X}} \pm \mathrm{S})$

\begin{tabular}{llll}
\hline Group & Number of animals (n) & $\begin{array}{l}\text { Jumping incubation } \\
\text { period (s) }\end{array}$ & Number of errors in 5min \\
\hline The control group & 12 & $82.92 \pm 39.19^{* *}$ & $5.83 \pm 1.85^{* *}$ \\
The model group & 12 & $39.17 \pm 22.64$ & $8.75 \pm 2.90$ \\
The low-dose LBP group & 12 & $89.83 \pm 43.13^{*}$ & $6.92 \pm 2.94$ \\
The high-dose LBP group & 12 & $95.75 \pm 49.26^{*}$ & $6.58 \pm 2.71^{*}$ \\
\hline
\end{tabular}

" Compared with the model group, $\mathrm{P}<0.01$; "Compared with the model group, $\mathrm{P}<0.05$

\section{Results of determination of LPO, LF and MAO-B contents in brain tissue}

The results of determination of LPO, LF and MAO-B contents in brain tissue are shown in Table 3-3. 45 days after modelling and drug administration, LPO, LF and MAO-B contents in brain tissue in the model group increased significantly, and compared with the model group, those in the drug administration groups decreased significantly.

Table 3: Results of determination of LPO, LF and MAO-B contents in brain tissue in aging mice $(\bar{X} \pm S, n=12)$

\begin{tabular}{|l|l|l|l|}
\hline Group & LPO $(\mathrm{nmol} / \mathrm{mg}$ tissue $)$ & LF fluorescence value & MAO-B $(\triangle \mathrm{A} / \mathrm{h})$ \\
\hline The control group & $10.58 \pm 1.93^{*}$ & $17.83 \pm 1.75^{* *}$ & $0.188 \pm 0.036^{*}$ \\
\hline The model group & $12.67 \pm 2.77$ & $19.58 \pm 1.44$ & $0.231 \pm 0.024$ \\
\hline The low-dose LBP group & $10.17 \pm 2.17^{*}$ & $13.17 \pm 1.99^{* *}$ & $0.194 \pm 0.022^{*}$ \\
\hline The high-dose LBP group & $9.83 \pm 1.99^{* *}$ & $15.83 \pm 1.95^{*}$ & $0.177 \pm 0.017^{*}$ \\
\hline
\end{tabular}

" Compared with the model group, $\mathrm{P}<0.01$; "Compared with the model group, $\mathrm{P}<0.05$ 
http://dx.doi.org/10.4314/ajtcam.v10i4.3

\section{Results of determination of immune organ mass index}

The results of determination of immune organ mass index are shown in Table 3-4. The thymus index in the model group decreased significantly; the thymus index and the spleen index in the high-dose LBP group and the low-dose LBP group rebounded significantly.

Table 4: Results of determination of thymus index and spleen index in aging mice $(\bar{X} \pm S, n=12)$

\begin{tabular}{|l|l|l|}
\hline Group & $\begin{array}{l}\text { Thymus index (mg/10g body } \\
\text { weight) }\end{array}$ & Spleen index (mg/10g body weight) \\
\hline The control group & $22.83 \pm 1.59^{* *}$ & $41.92 \pm 2.81$ \\
\hline The model group & $19.58 \pm 1.62$ & $40.33 \pm 2.99$ \\
\hline The low-dose LBP group & $26.33 \pm 1.37^{* *}$ & $46.42 \pm 3.53^{* *}$ \\
\hline The high-dose LBP group & $24.42 \pm 1.78^{* *}$ & $46.17 \pm 3.13^{* *}$ \\
\hline
\end{tabular}

" Compared with the model group, $\mathrm{P}<0.01$

\section{Discussion}

The metabolism theory of aging explains that aging is the result of body metabolism disorders. At continuous injection of D-galactose in animals within certain time, the galactose concentration increases. It will inevitably cause glucose metabolism disorder, thereby causing abnormal metabolism of heart, liver, kidney, brain and other important organs. Meanwhile, some products damaging cell membrane lipid are generated during the process that galactose is reduced into galactitol, such as the increases of lipid peroxidation and lipofuscin, finally leading to body aging (Cui et al., 2006), The results of this study show that the mice in the aging model group show significant aging symptoms in signs and the jumping test. The memory and learning abilities were in disorder, the incubation period reduced significantly, the number of errors increased significantly, and the number of electric shocks increased. After the mice drank LBP, compared with the aging model group, the memory and learning ability increased $(\mathrm{P}<0.05$ or $\mathrm{P}<0.01)$. LPO, LF and MAO-B contents in brain tissue relatively reduced. The above experimental results show that LBP has certain therapeutic effects on mouse brain function decline caused by D-galactose, providing the basis for the development of traditional Chinese medicine for the treatment of brain degenerative diseases.

Thymus, as the central immune organ, mainly mediates cellular immune response. The thymus index decreases, indicating that thymocyte proliferation and differentiation capacity and cellular immune function decrease. Spleen, as the second level of immune organ, is mainly involved in humoral immunity; it is the organ generated by antibodies, and it can remove senescent cells, microorganisms and other substances. Therefore, thymus and spleen are closely related with body aging. This study shows that LBP can delay the atrophy of thymus and spleen of aging mice, thereby preventing the decline of cellular immune and humoral immune functions, and has the effect of slowing aging to a certain extent. This experiment is simple and reliable in method, short in experiment period, small in individual difference and stable in repeatability (Zhao \& Yang., 1999; Xu et al., 2003).

\section{References}

1. Ayiguli Aihemaiti, Wang Ying, Han Haixia, et al. (2007). LBP extraction methods and comparative study on the content of polysaccharides in barbary wolfberry in the two different production areas. Xinjiang Agricultural Sciences, 44 (5): 724-728.

2. Cui Meizhi, Liu Hao, Li Chunyan. (2006). Establishment and evaluation of aging animal model [J]. Chinese Journal of 

http://dx.doi.org/10.4314/ajtcam.v10i4.3

Comparative Medicine, 16 (2): 118-121.

3. Kim, S. Y., Choi, Y. H., Kim, J., Kim, Y. C., \& Lee, H. S. (1997). New antihepatoxic cerebroside from Lycium chinese. Journal of Natural Product, 60:274-276.

4. Kim, S. Y., Lee, J. E., Kim, P. H., Lee, H. S., \& Kim, Y. C. (2000). LCC, a cerebroside from Lycium chinese, protects primary cultured rat hepatocytes exposed to galactoseamine. Phytotherapy Research, 14:448-451.

5. Leung, I., Li, W., Tso, M., \& Lam, T. (2001). Absorption and tissue distribution of zeaxanthin and lutein in rhesus monkeys after taking Fructus lycii (Gou Qi Zi) extract. Investigative Ophthalmology and Visual Science, 42:466-471.

6. Miao Mingsan. (2002). Effects of fructus schisandrae polysaccharides on aging model mouse. China Journal of Traditional Chinese Medicine and Pharmacy, 17 (3): 187.

7. Peng, X., \& Tian, G. (2001). Structural characterization of the glycan part of glycoconjugate LbGp2 from Lycium barbarum L. Carbohy drateResearch, 331(1):95-99.

8. State Pharmacopeia Committee. (2010).Pharmacopoeia of the People's Republic of China. Beijing: China Medical Science Press, 1:232-233.

9. Toyoda-Ono, T., Maeda, M., Nakao, M., Yoshimura, M., Sugiura- Tomimori, N., \& Fukami, H. (2004). 2-O (a-D-glucopyranosyl) ascorbic acid, a novel ascorbic acid analogue isolated from Lycium Friut. Journal of Agricultural and Food Chemistry, 52:2092-2096.

10. Xu Shuyun, Bian Rulian, Chen Xiu. (2003).Methodology of pharmacologic experiment ( $3^{\text {rd }}$ edition). Beijing : People's Medical Publishing House, 826-828.

11. Xu Zhi, Wu Guoming, Qian Guisheng, et al. (2003).Preliminary establishment of rat aging model. Acta Academiae Medicinae Militaris Tertiae, 25 (4): 312-315.a

12. Zhao Peng, Yang Yuying. (1999). Feasibility for subacute aging animal model with galactose. Chinese Journal of Food Hygiene, 11 (1): 52.

13. Zhou Jing, Li Guanghua. (2009). Study review on chemical composition and pharmacological effects of barbary wolfberry. Journal of Liaoning University of Traditional Chinese Medicine, 11 (6): 93-95. 